\title{
Rhabdomyolysis in Intensive Care Unit: More than One Cause
}

\author{
Puneet Saxena $^{1}$, Sahajal Dhooria ${ }^{2},{\text { Ritesh } \text { Agarwal }^{3}, \text { Kuruswamy Thurai Prasad }^{4} \text {, Inderpaul Singh Sehgal }}^{5}$
}

\begin{abstract}
Rhabdomyolysis is a serious medical condition, encountered in the intensive care unit (ICU). The etiology of rhabdomyolysis is often multifactorial. It leads to complications like acute kidney injury and life-threatening electrolyte abnormalities. A high index of suspicion and early institution of therapy is required to prevent complications and improve patient outcomes. Herein, we present the case of a young man with alcohol dependence who presented with fever and altered sensorium. He was found to have rhabdomyolysis and was managed successfully. We also discuss the common causes of rhabdomyolysis and a bedside approach to its management in the ICU.

Keywords: Delirium tremens, Renal failure, Rhabdomyolysis, Tropical myositis

Indian Journal of Critical Care Medicine (2019): 10.5005/jp-journals-10071-23238
\end{abstract}

\section{INTRODUCTION}

Rhabdomyolysis is a condition characterized by breakdown of skeletal muscles. This leads to the release of toxic intracellular contents into the systemic circulation. ${ }^{1}$ It is diagnosed by the presence of significantly elevated levels of creatine kinase (CK), and the excretion of myoglobin in the urine (myoglobinuria), which may impart a cola color to the urine. ${ }^{1}$ Rhabdomyolysis was initially described in the victims of war and natural disasters with crush injuries. However, the entity is frequently encountered in patients with a non-traumatic etiology of muscle breakdown. ${ }^{2}$ Chemical agents, specially the use of alcohol and illicit drugs, have frequently been implicated in triggering rhabdomyolysis. ${ }^{3-7}$ Rhabdomyolysis is often encountered in the intensive care setting and may have a multifactorial etiology. ${ }^{8}$ A high index of clinical suspicion and prompt initiation of treatment plays a vital role in the management of these cases. Herein, we describe the occurrence of rhabdomyolysis in a subject with chronic alcohol abuse who presented with high grade fever and altered sensorium.

\section{Case Description}

A 35-year-old male with alcohol dependence and a history of binge drinking a day prior, was admitted to a drug de-addiction center. He was treated with benzodiazepines and risperidone. Five days after initiating the treatment, he developed high-grade fever and altered behavior (irrelevant speech and conduct). There was no history of headache, vomiting, seizure or any focal neurological deficit. During hospitalization, he sustained injury to the right upper limb after a fall from the bed. His behavior progressively worsened over the next few days and by the tenth day of illness, he had stopped recognizing his relatives and had become disoriented to time and place.

At admission to the intensive care unit (ICU), he was agitated, un-cooperative and disoriented (time, place and person). He was febrile $\left(38^{\circ} \mathrm{C}\right)$ and had a heart rate of 120 beats/minute. His right upper limb showed erythema and induration suggestive of cellulitis. He was endotracheally intubated for airway protection. We considered the following diagnosis at admission to the ICU namely, delirium tremens, neuroleptic malignant syndrome, tropical pyomysositis or an infective illness common to tropics (leptospirosis, malaria or scrub typhus). \begin{tabular}{l}
\hline${ }^{1-5}$ Department of Pulmonary Medicine, Postgraduate Institute of \\
Medical Education and Research, Chandigarh, India
\end{tabular}

Corresponding Author: Inderpaul Singh Sehgal, Department of Pulmonary Medicine, Postgraduate Institute of Medical Education and Research, Chandigarh, India, Phone: 91-172-2746823, e-mail: inderpgi@outlook.com

How to cite this article: Saxena P, Dhooria S, Agarwal R, Prasad KT, Sehgal IS. Rhabdomyolysis in Intensive Care Unit: More than One Cause. Indian J Crit Care Med 2019;23(9):427-429.

Source of support: Nil

Conflict of interest: None

Initial investigations (Table 1) showed thrombocytopenia, hypernatremia, azotemia and raised aspartate aminotransferase (AST). Serum CK and urine myoglobin levels were elevated and the urine $\mathrm{pH}$ was 6.0. Cerebrospinal fluid was acellular with normal proteins and sugar. Computed tomography of the brain showed communicating ventriculomegaly. Ultrasound of the right upper limb revealed diffuse subcutaneous and muscle edema with no drainable collection suggesting cellulitis and myositis. Neuroleptic malignant syndrome was ruled out due to the absence of rigidity.

He was diagnosed to have delirium tremens with rhabdomyolysis, cellulitis and myositis of right arm, and acute kidney injury. The rhabdomyolysis was attributed to delirium tremens, hypernatremia, the use of risperidone and pyomyositis. Risperidone was discontinued. Blood culture grew methicillin resistant Staphylococcus aureus (MRSA) and injection vancomycin (2 grams/ day as continuous infusion) was started. During the hospital stay, the CK increased to a peak level of $35026 \mathrm{U} / \mathrm{L}$ on day 9 with a worsening of kidney function. Normal saline was administered to manage rhabdomyolysis with a goal of maintaining the urine output at 200-300 mL/hour. The hospital course was complicated by worsening of hypernatremia (up to $180 \mathrm{mEq} / \mathrm{L}$ ) that was managed with administration of free water. The hypernatremia was possibly due to intravascular dehydration as a consequence of rhabdomyolysis and infective myositis. The subject gradually improved with treatment. His mental status improved, the urine $\mathrm{pH}$ increased to $>6.5$, and the renal functions normalized. The subject was shifted to ward on day 28 of his illness. He was subsequently discharged after 6 weeks with no residual deficit. At 4 months of

() The Author(s). 2019 Open Access This article is distributed under the terms of the Creative Commons Attribution 4.0 International License (https://creativecommons. org/licenses/by-nc/4.0/), which permits unrestricted use, distribution, and non-commercial reproduction in any medium, provided you give appropriate credit to the original author(s) and the source, provide a link to the Creative Commons license, and indicate if changes were made. The Creative Commons Public Domain Dedication waiver (http://creativecommons.org/publicdomain/zero/1.0/) applies to the data made available in this article, unless otherwise stated. 
Table 1: Complete blood count, biochemistry and other investigations at admission to intensive care unit

\begin{tabular}{|c|c|}
\hline Parameter & Value \\
\hline Hemoglobin (g/dL) & 16.6 \\
\hline Total leukocyte count (cells $/ \mathrm{mm}^{3}$ ) & 7000 \\
\hline Platelets (per $\mathrm{mm}^{3}$ ) & 107,000 \\
\hline Sodium (mEq/L) & 164 \\
\hline Potassium (mEq/L) & 4.4 \\
\hline Calcium_corrected for albumin (mg/dL) & 8.7 \\
\hline Phosphorus (mg/dL) & 2.6 \\
\hline Uric acid (mg/dL) & 7.7 \\
\hline Urea (mg/dL) & 102 \\
\hline Creatinine (mg/dL) & 1.7 \\
\hline Aspartate aminotransferase (U/L) & 493 \\
\hline Alanine aminotransferase (U/L) & 81 \\
\hline Creatine kinase $(\mathrm{U} / \mathrm{L})$ & 7056.2 \\
\hline Urine myoglobin (ng/mL) & 836 \\
\hline Human immunodeficiency virus (ELISA) & Negative \\
\hline \multicolumn{2}{|l|}{ Cerebrospinal fluid examination } \\
\hline Cells (per high-power field) & 0 \\
\hline Proteins (mg/dL) & 19 \\
\hline Glucose (mg/dL) & 111 \\
\hline Blood culture & $\begin{array}{l}\text { Methicillin resistant Staphylococcus } \\
\text { aureus }\end{array}$ \\
\hline Malaria $^{*}$ & Negative \\
\hline Leptospira** & Negative \\
\hline Scrub typhus ${ }^{\#}$ & Negative \\
\hline
\end{tabular}

*Malaria was tested with examination of peripheral smear as well as "SD BIOLINE Malaria Pf/Pv test" - a rapid immunochromatographic test for simultaneous detection of antibodies against Plasmodium falciparum and Plasmodium vivax in blood (Alere Medical Pvt. Ltd.)

**Leptospira was tested with "SD ELISA Kit" (Enzyme immunoassay for the detection of IgM antibodies to Leptospira - Standard Diagnostics, Inc.; IgM ELISA)

\#Scrub typhus was tested with "Scrub Typhus Detect ${ }^{\mathrm{TM}}$ IgM ELISA System" (InBios International Inc.)

follow-up, he was doing well and continued to abstain from alcohol consumption.

\section{Discussion}

Rhabdomyolysis is a medical emergency that requires high clinical suspicion and an early treatment. The index case highlights that rhabdomyolysis may be multifactorial and may resolve completely with appropriate management.

Rhabdomyolysis has several causes that can be broadly categorized as hypoxic, physical, chemical and biologic (Table 2). Chemical causes are responsible for most of the cases of rhabdomyolysis. Apart from the intrinsic factors (electrolyte abnormalities), many extrinsic factors (alcohol consumption, psychiatric medications and illicit drug use) are known to cause rhabdomyolysis. Physical causes of rhabdomyolysis are the most common and can be due to either the external (direct trauma) or internal (voluntary and involuntary) factors. ${ }^{1,2,5,9}$ However, several of these factors may contribute in a given case. ${ }^{3}$ This was highlighted in the index case where multiple factors (trauma to the limb, delirium tremens, use of risperidone, hypernatremia) were the contributing factors. Also, chronic alcohol consumption predisposes an individual to rhabdomyolysis due to electrolyte abnormalities, malnutrition and limited energy stores.
The symptoms of rhabdomyolysis are nonspecific and the diagnosis may be overlooked especially in absence of classical triad of myalgia, muscle weakness and dark colored urine. Diagnosis is established by elevated CK levels (usually $>10$ times the upper limit of normal) and myoglobinuria that is attributed to muscle destruction and its release into the circulation. ${ }^{2}$ Management of rhabdomyolysis primarily involves preventing further muscle injury, and potential complications (cardiac arrhythmias and acute renal failure). ${ }^{1,5}$ This is especially important when multiple causes of rhabdomyolysis coexist, as in the index case. The index case was managed with lorazepam (delirium tremens), antibiotics (pyomyositis), and administration of free water (hypernatremia), thus addressing all the likely factors contributing to muscle destruction. After eliminating the precipitating cause of rhabdomyolysis, the management is aimed at preventing renal insult. Acute kidney injury may develop in up to $60 \%$ patients with rhabdomyolysis. ${ }^{9}$ Use of aggressive fluid resuscitation (with isotonic saline), is recommended to prevent further renal damage. The aggressive fluid management is continued till the resolution of rhabdomyolysis as evidenced by falling CK levels or when oliguric AKI precludes further fluid infusion, as in the index case. ${ }^{1,9}$ In those with oliguric or anuric kidney injury, an early renal support therapy with hemodialysis 
Table 2: Causes of nontraumatic rhabdomyolysis

\begin{tabular}{|c|c|c|}
\hline Mechanism & Extrinsic causes & Intrinsic causes \\
\hline \multirow[t]{3}{*}{ Hypoxic } & Carbon monoxide exposure & Compartment syndrome \\
\hline & Cyanide exposure & $\begin{array}{l}\text { Vascular insufficiency (thrombosis, compression, } \\
\text { vasculitis, sickle cell disease, shock states) }\end{array}$ \\
\hline & & Immobilization (prolonged surgery, coma) \\
\hline \multirow[t]{6}{*}{ Physical } & Hypothermia & Prolonged and/or extreme exertion \\
\hline & Hyperthermia & Acute asthma \\
\hline & & Seizures \\
\hline & & Agitation (delirium tremens) \\
\hline & & Neuroleptic malignant syndrome \\
\hline & & Malignant hyperthermia \\
\hline \multirow[t]{3}{*}{ Chemical } & Alcohol & Hypokalemia \\
\hline & $\begin{array}{l}\text { Other illicit drugs like cocaine, amphetamines, } \\
\text { heroin }\end{array}$ & $\begin{array}{l}\text { Hypophosphatemia, hypocalcemia, } \\
\text { hyponatremia, hypernatremia }\end{array}$ \\
\hline & $\begin{array}{l}\text { Prescription drugs (statins, antipsychotics, } \\
\text { valproate, propofol, diphenhydramine and } \\
\text { antimicrobials like trimethoprim-sulfamethoxazole, } \\
\text { quinolones, amphotericin B }\end{array}$ & \\
\hline \multirow[t]{4}{*}{ Biological } & Infective myositis (bacterial, viral or parasitic) & Dermatomyositis/polymyositis \\
\hline & Toxins (snake bite, insect stings and others) & $\begin{array}{l}\text { Endocrinopathies like adrenal insufficiency, } \\
\text { hypothyroidism, hyperaldosteronism and } \\
\text { diabetic ketoacidosis }\end{array}$ \\
\hline & & Hyperosmolar state \\
\hline & & Genetic inborn errors of metabolism \\
\hline
\end{tabular}

should be considered. Alkalinization of urine and forced diuresis with diuretics or mannitol, are not preferred currently. ${ }^{1,10}$

In conclusion, rhabdomyolysis is a medical emergency that may have multifactorial etiologies. Identifying and correcting each component is important to prevent further muscle injury and organ failure. Early identification and institution of treatment is the cornerstone to prevent AKI and other serious complications of rhabdomyolysis.

\section{References}

1. Zimmerman JL, Shen MC. Rhabdomyolysis. Chest 2013;144:10581065.

2. Zutt R, van der Kooi AJ, Linthorst GE, Wanders RJ, de Visser M. Rhabdomyolysis: review of the literature. Neuromuscul Disord 2014;24:651-659.

3. Melli G, Chaudhry V, Cornblath DR. Rhabdomyolysis: an evaluation of 475 hospitalized patients. Medicine (Baltimore) 2005;84:377-385.
4. Barbano B, Sardo L, Gasperini ML, Gigante A, Liberatori M, Di Lazzaro GG, et al. Drugs and Rhabdomyolysis: From Liver to Kidney. Curr Vasc Pharmacol 2015;13:725-737.

5. Cervellin G, Comelli I, Benatti M, Sanchis-Gomar F, Bassi A, Lippi G. Non-traumatic rhabdomyolysis: Background, laboratory features, and acute clinical management. Clin Biochem 2017;50:656-662.

6. Curry SC,Chang D, ConnorD.Drug-and toxin-induced rhabdomyolysis. Ann Emerg Med 1989;18:1068-1084.

7. Richards JR. Rhabdomyolysis and drugs of abuse. J Emerg Med 2000;19:51-56.

8. Shapiro ML, Baldea A, Luchette FA. Rhabdomyolysis in the intensive care unit. J Intensive Care Med 2012;27:335-342.

9. Bosch X, Poch E, Grau JM. Rhabdomyolysis and acute kidney injury. N Engl J Med 2009;361:62-72.

10. Brown CV, Rhee P, Chan L, Evans K, Demetriades D, Velmahos GC. Preventing renal failure in patients with rhabdomyolysis: do bicarbonate and mannitol make a difference? J Trauma 2004;56:11911196. 